\title{
Variables sociocognitivas y su relación con la actividad física en estudiantes universitarios chilenos \\ Sociocognitive variables and their relationship with physical activity in chilean university students

\author{
*Javier Andrés M ella-N orambuena, **Gabriela N azar Carter, ***Fabiola Sáez-Delgado, **Claudio Bustos \\ $\mathrm{N}$ avarrete, $* * * *$ Yaranay López-Angulo, $* *$ Rubia Cobo Rendón \\ *U niversidadTécnica Federico Santa M aría (Chile), **U niversidad de Concepción (Chile), ***U niversidad Católica de la \\ Santísima Concepción (Chile), ****U niversidad Santo Tomás (Chile).
}

\begin{abstract}
Resumen: Los factores que influyen en los niveles de actividad física son muchos, sin embargo, es necesario identificar aquellos que pueden ser modificables, entre ellos están las variables sociocognitivas. El objetivo general de esta investigación fue anal izar la relación entre el autoconcepto físico, motivación, autoeficacia y percepción de barreras paralaactividad física, y el bienestar subjetivo con el nivel de actividad física en estudiantes universitarios. Se utilizó un diseño predictivo transversal y participaron 362 estudiantes de Educación Superior de una región del centro sur de Chile. Para responder a los objetivos se utilizó un Modelo de Ecuaciones Estructurales, específicamente el modelo Probit adecuado para regresiones ordinales. Los resultados mostraron que: (1) la autoeficacia para la práctica de actividad física, la falta de tiempo, fal ta de voluntad, falta de habilidad, el autoconcepto de condición física y el autoconcepto físico de fuerza predijeron significativamente el nivel de práctica de actividad física; (2) el nivel de actividad física media la relación entre las variables sociocognitivas y la afectividad positiva. Es posible concluir que lasvariables sociocognitivas influyen en losniveles de actividad físicade estudiantes universitarios y estaúltimainfluye en su bienestar.
\end{abstract}

Palabras claves: Actividad física, motivación, autoconcepto, autoeficacia, bienestar, percepción de barreras, universitarios, modelo estadístico.

\begin{abstract}
The factors that influence physical activity levels are many, however, it is necessary to identify those that can be modified, among them are the sociocognitive variables. The general objective of this research was to analyze the relationship between physical self-concept, motivation, self-efficacy and perception of barriers to physical activity, and subjective wellbeing with the level of physical activity in university students. A cross-sectional predictive design was used and 362 students of $\mathrm{H}$ igher Education from a region of southern central Chile participated. To respond to the objectives, a M odel of Structural Equations was used, specifically the Probit model suitable for ordinal regressions. The results showed that: (1) self-efficacy for the practice of physical activity, lack of time, lack of will, lack of ability, self-concept of physical condition and physical selfconcept of strength significantly predicted the level of practice of physical activity; (2) the level of physical activity mediatesthe relationship between sociocognitive variables and positive affectivity. It is possible to conclude that sociocognitive variables influence the levels of physical activity of university students and the latter influences their well-being.
\end{abstract}

Keywords: Physical activity, motivation, self-concept, self-efficacy, well-being, barrier perception, university students, staistical model.

\section{Introducción}

La práctica regular de actividad física (en adelante $\mathrm{AF})$, ha sido asociada consistentemente con diversos beneficios, físicos, psicológicos, cognitivos, sociales, emocionales (Warburton \& Bredin, 2017), y desempeña un rol protagónico en la prevención de algunas enfermedadescrónicas no transmisibles(O MS, 2010). Los estudiantes universitarios son considerados una pobla ción clave para la promoción de laAF, puesto que están

Fecha recepción: 16-02-20. Fecha de aceptación: 05-10-20 Javier Andrés Mella Norambuena

javier.mellan@usm.cl en un período crítico y vulnerable de cambio de peso corporal y adopción de un estilo de vida (Kim, Lumpkin, Lochbaum, Stegemeier \& Kitten, 2018). El nivel deAF realizado durante el último año universitario, se considera uno de los predictores más influyentes de la AF post-universitaria (Corella, Rodríguez-M uñoz, Abarca Sos \& Zaragoza, 2018).

Sin embargo, hay poca claridad en los factores que determinan los niveles de AF, siendo necesario más investigaciones que permitan examinar las variables que dificultan u obstaculizan alcanzar niveles recomendados (Sevil, Práxedes, Zaragoza, Del Villar \& GarciaGonzález, 2017). Aunque las variables que influyen en 
la AF son diversas, en psicología de la salud, se ha centrado la investigación en variables sociocognitivas (en adelanteVSC), puesto que son, por una parte, los factores más proximales al comportamiento y por otra, son susceptibles de modificar, al contrario de otras varia bles, como las sociodemográficas (Mirzaei-Alavijeh et al., 2018).

LasVSC del cambio conductual en salud relaciona das a la AF con evidencia teórica y empírica (Lubans, O kely, M organ, Cotton, Puglisi \& M iller, 2012; Majeed, Jabbar \& Jun, 2017; M irzaei-Alavijeh et al., 2018) esta blece que: (1) la motivación, (2) la autoeficacia, (3) el autoconcepto, (4) la percepción de barreras influyen directamente en laAF y esta a su vez influye en el bienestar subjetivo (Zhang, $\mathrm{He} \&$ Chen, 2020).

La O rganización M undial de la Salud define la AF como todo movimiento corporal generado por los músculosesqueléticos, queconllevan aun consumo de energía (O MS, 2010). Respecto del bienestar subjetivo, desde laTeoría de Diener (1984), se define como la val oración individual que hacen una persona de su calidad de vida desde una construcción tridimensional (satiffacción con la vida, afecto positivo y afecto negativo). La investiga ción ha mostrado relación positiva y significativa entre la práctica de AF y el bienestar (Pereira, Fernandez, Cruz \& Santiesteban, 2018; Zhang, et al ., 2020).

La motivación, desde la Teoría de la Autodeterminación es definida como el grado en que cada persona ejecuta sus acciones en un continuo de autodeterminación que revela tipos de motivación de acuerdo con estilos de regulación en 6 niveles que van desde la amotivación (ausencia de regulación), la motivación extrínseca (regulación externa, introyectada, identificada e integrada), hasta la motivación autónoma (regulación intrínseca) (Deci \& Ryan, 2000). Se ha demostrado que las formas de motivación más autodeterminadas ejercen influencia en los niveles de práctica deAF (Chanal, Cheval, Courvoisier \& Paumier, 2019).

La autoeficacia para la AF desde la Teoría Social Cognitiva es definida como la creencia sobre la propia capacidad para mantener la práctica de AF en distintas situaciones (Marcus, Selby, Niaura \& Rossi, 1992). Es una de las teorías más utilizadas en el estudio del cambio de comportamiento en salud (Strecher, M CEvoy, Becker \& Rosenstock, 1986) y considerada un predictor de la conducta de AF (W illiams \& French, 2011).

El autoconcepto físico es definido como una autoevaluación individual de atributos en el dominio físico que real izala persona y el modelo teórico más acep- tado lo describe como un constructo tetradimensional (Fox \& Corbin, 1989): condición física, competencia deportiva, apariencia física y fuerza. Se ha evidenciado que el autoconcepto físico es determinante de la AF (Lemoyne, Valois \& Guay, 2015).

La percepción de barreras se define como los obstá culos que hacen que sea difícil realizar comportamientos de AF (Ramírez-Vélez, Triana-Reina, Carrillo, \& Ramos-Sepúlveda, 2016), y han demostrado relación con la prácticadeAF en universitarios (M artínez-Lemos, Puig \& García-García, 2014).

Estas cuatro variables se han estudiado por el reconocimiento de su efecto sobre la conducta humana, sin embargo, las investigaciones previas presentan limitaciones en cuanto estas: (a) se han centrado principalmente en estudios de al cance descriptivo-correlacional (Sibley \& Bergman, 2016); (b) y los estudios predictivos existentes sólo consideran algunas de las VSC que han mostrado evidencia de ser predictoras de laAF, presentando, además, baja calidad metodológica (Young, Plotnikoff, Collins, Callister \& M organ, 2014). En definitiva, no se ha encontrado un estudio que incorpore en Ios análisis todas las VSC que han mostrado cambios positivos en la AF. En consecuencia, se enfatiza la necesidad de proponer y examinar modelos con VSC para explicar los niveles de AF.

Por lo anterior, este estudio estableció como objetivo general estudiar la asociación entre la motivación, el autoconcepto físico, la percepción de barreras para la AF, la autoeficacia, y el bienestar subjetivo con el nivel de AF. Específicamente: (1) analizar en qué medida las VSC (motivación, autoeficacia para laAF, autoconcepto físico, percepción de barreras para la AF) predicen el nivel de AF; y (2) analizar el efecto de mediación del nivel de AF entre lasVSC y el bienestar subjetivo.

\section{Método}

Para estudiar la asociación entre las variables de esta investigación se utilizó un diseño predictivo transversal (Ato, López \& Benavente, 2013).

\section{Instrumentos}

Actividad Física: Cuestionario Internacional de Actividad Física (IPAQ) versión corta que tiene 7 ítems y entrega información acerca del tiempo que la persona dedica en actividades de intensidad moderada y vigorosa, así como caminar y estar sentado. Evalúa tres características de la AF: intensidad (leve, moderada o vigorosa), frecuencia (días por semana) y duración (tiempo 
por día). La actividad semanal se registra en METS (M etabolic Equivalent of Task o U nidades de Índice Metabólico) por minuto y semana (Carrera, 2017; Serón, Muñoz \& Lanas, 2010).

Bienestar Subjetivo: Se utilizó el Cuestionario de Satisfacción con la Vida (Álvarez, Briceño, Álvarez, Abufhele \& Delgado, 2018). Después de leer la decla ración «En qué medida usted está de acuerdo con... », los participantes respondieron 5 ítems ( $\alpha=.82$; ejemplo: «estoy satiffecho con mi vida»). También se usó la Escala de afecto positivo y negativo (D ufey \& Fernandez, 2012), tiene 20 ítems (10 ítems por factor) que siguen el enunciado "Cómo se ha sentido en el último mes, incluyendo el día de hoy.. » y miden afectividad positiva ( $\alpha=$.80; ejemplo «interesado/ a por las cosas», y afectividad negativa ( $\alpha=.60$; ejemplo «angustiado/ a»).

Autoeficacia para la práctica de AF: Se utilizó el Cuestionario de Autoeficacia para el ejercicio (Delga do, Zamarripa, De LaCruz, Cantú-Berrueto \& Álvarez, 2017). Después de leer la declaración «Q ué tan confia do está para ser físicamente activo. .», los participantes respondieron 5 ítems ( $\alpha=.81$; ejemplo: Cuando estoy cansado).

Motivación para laAF: Se usó el Cuestionario de la Regulación de la Conducta en el Ejercicio (BREQ -3) (González-Cutre, Sicilia \& Fernández, 2010). Este instrumento tiene 22 ítems ( 4 factores de 4 ítems y 2 factores de 3 ítems) que se responden después de leer la declaración «yo hago ejercicio físico... ) y mide la Regulación intrínseca ( $\alpha=$.87; ejemplo: Porque creo que el ejercicio es divertido), regulación integrada ( $\alpha$ $=$.87; ejemplo: Porque está de acuerdo con mi forma de vida), regulación identificada ( $\alpha=.66$; ejemplo: Porque valoro los beneficios que tiene el ejercicio físico), regulación introyectada ( $\alpha=72$; ejemplo: Porque me siento culpable cuando no lo practico), regulación externa ( $\alpha=78$; ejemplo: porque los demás me dicen que debo hacerlo), y desmotivación ( $\alpha=70$; ejemplo: No veo por qué tengo que hacerlo).

Autoconcepto físico: se utilizó el Cuestionario de Autoconcepto Físico (CAF) (M oreno \& Cervelló, 2005). Este instrumento tiene 30 ítems (1 factor con 8 ítems, 2 factores con 6 ítems y dos factores con 5 ítems) que se responden después de leer la declaración «C uando realizo actividad física... » y mide condición física ( $\alpha=$ .80; ejemplo: siempre mantengo una excelente condición y forma física), apariencia física ( $\alpha=.70$; ejemplo: Siempre estoy satisfecho/ a de cómo soy físicamente), competencia percibida ( $\alpha=.83$; ejemplo: Soy muy bueno/ a en casi todos los deportes), fuerza ( $\alpha=.63$; ejemplo: mis músculos son tan fuertes como los de la mayoría de las personas de mí mismo sexo), autoestima $(\alpha=.74$; ejemplo: Creo que no estoy entre los/ as más capaces cuando se trata de habilidad deportiva).

Percepción de Barreras para la Práctica de la Actividad Física: Se usó el Cuestionario sobre barreras para mantenerse activo (Rubio-Henao et al. , 2015). Esteinstrumento tiene 18 ítems ( 3 ítems para cada factor) que se responden después de leer la declaración «Indique marcando con un círculo qué probabilidad tiene usted de decir las siguientes excusas... » y mide fal ta de tiempo ( $\alpha=$.79; ejemplo: Mi día es tan ocupado que no creo que pueda apartar tiempo para realizar actividad física), influencia social ( $\alpha=.79$; ejemplo: A ninguno de mis familiares 0 amigos les gusta realizar actividad física, por esto no hago ejercicio.), falta de energía ( $\alpha$ = .77; ejemplo: Estoy muy cansado después del trabajo como para hacer ejercicio), falta de voluntad ( $\alpha=.77$; ejemplo: he estado pensando en empezar a hacer ejercicio, pero no he sido capaz de dar el primer paso), miedo a lastimarse ( $\alpha=.62$; ejemplo: hacer ejercicio puede ser riesgoso a mi edad.), falta de habilidad ( $\alpha=$ .70; ejemplo: no hago ejercicio lo suficiente porque nunca he aprendido ningún deporte).

\section{Participantes}

La muestra fue no probabilística por conveniencia, con un total de 362 estudiantes de seis U niversidades del Sur de Chile. Específicamente 248 (65,8\%) hombres y $114(31,5 \%)$ mujeres, con una media de edad de $21,45$ años ( $D E=3,8)$. El criterio de inclusión fue ser estudiante universitario de cualquier carrera 0 año de estudio. Los criterios de exclusión fueron: (1) presentar alguna indicación médica que le impida el desarrollo de AF y (2) presentar alguna discapacidad física que le impida el desarrollo deAF.

\section{Procedimiento de recolección de datos y consi- deraciones éticas}

Se solicitó autorizaciones a directivos de las faculta des de las universidades participantes. También se solicitó la autorización a jefes de carrera y docentes y se les pidió tiempo de sus clases para la aplicación del instrumento. Antes de la aplicación de instrumentos, se realizó el procedimiento de firma de consentimiento informado alos participantes. El formulario de consentimiento fue revisado y aprobado previamente por el Comité de Ética y Bioética del Departamento de Psicología de la U niversidad de Concepción. Luego de recogida la información, sellenó la base de datos en M icrosoft Excel 
y se procedió a realizar los análisis de datos correspondientes.

\section{Procedimiento de análisis de datos}

Se anal izó la consistencia interna de cada escala por medio del Alfa de Cronbach. Despúes, se analizó de forma descriptiva la muestra, obteniendo las medidas detendenciacentral paracadavariable. Luego, segraficó la relación entre las variables para verificar la linealidad de la relación.

Para poder responder al objetivo 1 se ocupó un modelo ordinal probit (MDROP), que usa la normal acumulativa en lugar de la logísticay es pertinente cuando una variable de resultado es binaria u ordinal, es decir tiene categorías (Breen, Karlson \& Holm, 2018), en este caso, la variable predicha es el nivel de AF y tiene 3 niveles: bajo, moderado y alto. Se incluyó un modelo de regresión probit, ya que permite probar los supuestos de la regresión probit, acción que se da por sentada, o simplemente no se verifica en los modelos SEM. Esto nos permite contar con un resultado robusto, que sirve de base para el modelo posterior.

Para el objetivo 2 se usó un Modelo de Ecuaciones Estructurales (SEM por sus siglas en inglés). Se uso el MDRO P en vez del ordinal logit que es más usual, porque permite hacer homólogos los resultados entre la regresión por sí sola y SEM. Los análisis se efectuaron utilizando el software estadístico R, versión 3.6.1.

\section{Resultados}

Los resultados se muestran de acuerdo con los objetivos establecidos. Previamente, se presentan los análisis descriptivos de las variables.

\section{Resultados del análisis descriptivo de las va- riables}

Para la variable AF, del total de participantes ( $n=$ 362), 28 estudiantes (8\%) presentaron un nivel bajo de AF, 145 estudiantes (40\%) se encontraron en nivel moderado y 189 estudiantes (52\%) se encontraron en nivel alto. En la tabla 1 se presentan los resultados del análisis descriptivo para cada una de lasVSC de este estudio. Se puede obser var que las variables: satisfacción con la vida, afectividad positiva, autoeficacia para la AF, regulación (intrínseca, integrada e identificada), y autoconcepto físico, presenta una media superior al punto central teórico en cada escala. También se observó que la percepción de las diferentes barreras hacia la práctica de AF ninguna superó la media de la escala.

\begin{tabular}{lcccc} 
Tabla 1. & & & & \\
Descriptivos de las variables & Min & Máx & M & DE \\
\hline & 1 & 7 & 5.06 & 1.21 \\
\hline Bienestar subjetivo & 0 & 4 & 2.29 & 0.78 \\
Satisfacción con la vida & 0 & 4 & 1.27 & 0.84 \\
Afectividad positiva & & & & \\
Afectividad negativa & 0 & 4 & 2.85 & 0.87 \\
Autoeficacia paralaActividad Física & & & & \\
Autoeficacia para laActividad Física & 0 & 4 & 2.7 & 1.22 \\
Motivación & 0 & 4 & 2.06 & 1.24 \\
Regulación intrínseca & 0 & 4 & 2.67 & 1.16 \\
Regulación integrada & 0 & 4 & 1.15 & 0.93 \\
Regulación identificada & 0 & 4 & 0.62 & 0.83 \\
Regulación introyectada & 0 & 4 & 0.8 & 0.82 \\
Regulación externa & & & & \\
Desmotivación & 0 & 3 & 1.33 & 0.91 \\
Percepción de Barreras & 0 & 3 & 0.81 & 0.71 \\
Falta de tiempo & 0 & 3 & 1.36 & 0.91 \\
Influencia social & 0 & 3 & 1.29 & 0.93 \\
Falta de energía & 0 & 3 & 0.5 & 0.63 \\
Falta de voluntad & 0 & 3 & 0.53 & 0.68 \\
Miedo a lastimarse & 0 & 3 & 0.72 & 0.66 \\
Falta de habilidad & & & & \\
Falta de recur sos & 1 & 4 & 2.48 & 0.7 \\
Autoconcepto físico & 1 & 4 & 2.43 & 0.66 \\
Condición física & 1 & 4 & 2.42 & 0.72 \\
Apariencia física & 1 & 4 & 2.42 & 0.61 \\
Competencia percibida & 1 & 4 & 2.8 & 0.68 \\
\hline Fuerza & & & &
\end{tabular}

\section{Resultados Objetivo 1}

Al realizar el análisis del modelo, de prueba de razón de verosimilitud de $X^{2}$, mostró que el conjunto de VSC predice significativamente el nivel de AF $X^{2}(21)=173.78 ; p<.001$. Específicamente, el modelo ordinal mostró que un mayor nivel de AF se predice significativamente por: (1) un mayor nivel de autoeficacia para la práctica deAF $(b=0.44, p<.001)$; (2) un ma yor Autoconcepto de Condición física $(b=0.55, p<$ .01); (3) menor falta de tiempo ( $b=-0,31, p<.05)$; (4) menor falta de voluntad $(b=-0.43, p<0.001) ;(5)$ mayor falta de habilidad $(b=0.37, p<.05)$; (6) y menor autoconcepto físico de fuerza $(b=-0.38, p<.05)$ (ver tabla 2).

\begin{tabular}{|c|c|c|c|c|c|}
\hline & Sigla & Estimador & Std. Error & $z$ value $\operatorname{Pr}$ & $(>|z|)$ \\
\hline \multicolumn{6}{|l|}{ Variables sociodemográficas } \\
\hline Sexo (Mujer) & & -0.26 & 0.16 & -1.60 & 011 \\
\hline & \multicolumn{4}{|c|}{ Autoeficacia para la práctica de actividad física } & 0.02 \\
\hline Autoeficacia para la práctica de AF & AAF & $0.44 * * *$ & 0.11 & 4.04 & 0.00 \\
\hline \multicolumn{6}{|l|}{ Motivación } \\
\hline Regulación intrínseca & RINTRIN & 0.14 & 0.10 & 1.32 & 0.19 \\
\hline Regulación integrada & RINTEG & -0.08 & 0.12 & -0.67 & 0.50 \\
\hline Regulación identificada & RIDENT & 0.16 & 0.11 & 1.36 & 0.17 \\
\hline Regulación introyectada & RINTRO & 0.03 & 0.10 & 0.25 & 0.81 \\
\hline Regulación externa & REXTER & 0.16 & 0.11 & 1.48 & 0.14 \\
\hline Desmotivación & DESMOT & -0.02 & 0.11 & -0.20 & 0.84 \\
\hline \multicolumn{6}{|c|}{ Percepción de barreras para la práctica de actividad física } \\
\hline Falta de tiempo & FTIEMPO & $-0.31 * *$ & 0.12 & -2.45 & 0.01 \\
\hline Influencia Social & INFSOC & 0.12 & 0.14 & 0.83 & 0.41 \\
\hline Falta de energía & FENERG & 0.25 & 0.14 & 1.81 & 0.07 \\
\hline Falta de voluntad & FVOLUN & $-0.43 * * *$ & 0.11 & -3.87 & 0.00 \\
\hline Miedo a lastimarse & MIEDO & -0.10 & 0.15 & -0.66 & 0.51 \\
\hline Falta de habilidad & FALHAB & $0.37 *$ & 0.16 & 2.37 & 0.02 \\
\hline Falta de recursos & FALREC & -0.9 & 0.14 & -1.38 & 0.17 \\
\hline \multicolumn{6}{|l|}{ Autoconcepto físico } \\
\hline De la condición física & AUTOCF & $0.56 * *$ & 0.20 & 2.80 & 0.01 \\
\hline De la apariencia & AUTOAP & -0.11 & 0.15 & -0.79 & 0.43 \\
\hline De la competencia percibida & AUTOCOMP & 0.05 & 0.17 & 0.27 & 0.78 \\
\hline De la fuerza & AUTOF & $-0.38 * *$ & 0.16 & -2.46 & 0.01 \\
\hline De la autoestima & AUTOAU & 0.13 & 0.15 & 0.86 & 0.39 \\
\hline
\end{tabular}

En los modelos de regresión logística ordinal probit hay una suposición importante que pertenece a las pro- 
babilidades ordinales. De acuerdo con este supuesto, los parámetros no deberían cambiar para diferentes categorías (Xu, Bauldry \& Fullerton, 2019). Los resultados mostraron que el supuesto se cumple. Es decir, que las VSC influyen de la misma forma en el paso de los niveles 1 a 2 y de los niveles 2 a 3 deAF.

Una vez que se decidió mantener el modelo probit ordinal original, para calcular el nivel de ajuste de este modelo, se analizaron cuántos casos se predicen de manera correcta. Al observar la tabla 4, el modelo en teoría predice un $68,7 \%$ de casos de forma correcta. Sin embargo, este valor suele estar sobreestimado. Además, es importante destacar que de los tres niveles de AF el modelo en teoría predice mejor para los niveles 3 y 2 (alto y moderado), pero en el caso del nivel 1 (bajo), no es un buen predictor teórico. Esto se observa en la tabla 3, dado que en el nivel 1, de los 28 estudiantes observados en este nivel, el modelo sólo predice a 5 (18\%). En el caso del nivel 2, de los 145 estudiantes observados en este nivel, el modelo predice correcta mente a 95 (66\%). Finalmente, en el nivel 3, el número de estudiantes observados es de 189 y el modelo predice a 149, por tanto, en esta categoría, el modelo logra la mejor predicción (79\%).

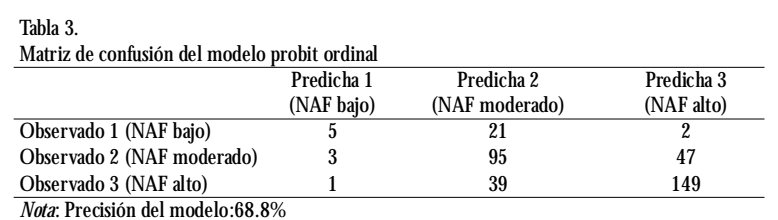

Finalmente, para asegurar el porcentaje de predicción del Modelo probit ordinal, se calculó el optimismo (diferencia entre lo predicho dentro de la muestra haciala población), utilizando un procedimiento basado en bootstrap (Efron, 1983), que mostró ser de 0.26 (calculado con 1000 remuestras). Por tanto, un estimador más realista de la precisión sería la diferencia entre el valor predicho teórico y el optimismo $(0.69-0.26=0.43)$. Se debe recordar que el modelo funciona mejor para distinguir entre los niveles moderados y altos (2 y 3 ), no entre bajos y el resto de los niveles.

\section{Resultados Objetivo 2}

El objetivo 2 de este estudio fue analizar el efecto de mediación del nivel deAF entre lasVSC y el bienestar subjetivo. Para responder a este objetivo se analiza ron tres coeficientes. El coeficiente «a» que representa la relación entre lasVSC y el nivel deAF; el coeficiente «b» que representa la relación entre el nivel deAF y las variables de bienestar (satisfacción con la vida y afecti- vidad positiva y negativa); y el coeficiente «(» que representa la relación entre las VSC y el bienestar no mediado por el nivel de AF. A continuación, se presentan los resultados de los análisis de cada uno de estos coeficientes.

Primero se revisó el efecto que tiene cada una de las VSC sobre el nivel deAF (coeficiente a) porque, es útil para entender el sentido de las relaciones y su fuerza. Esto se analizó en el modelo anterior desarrollado para el objetivo 1 (ver Tabla 3), evidenciando que las VSC con un efecto significativo sobre la AF fueron la autoeficacia parala práctica deAF, autoconcepto de condición física, falta de tiempo, falta de voluntad, falta de habilidad, y autoconcepto físico de fuerza.

En segundo lugar, se analizó la relación entre el nivel deAF y las variables de bienestar (satisfacción con la vida, afectividad positiva y afectividad negativa), es decir el coeficiente «b». Como se observa en la tabla 4, el nivel de AF tuvo un efecto significativo sólo en la escala de afectividad positiva. Posterior mente se analizaron los efectos directos o coeficiente «c» que corresponde a la relación entre las VSC y el bienestar, cuyo resultado indicó relaciones significativas entre las variables autoconcepto físico de la apariencia y satisfacción con la vida $(0.49, p<.01)$, autoconcepto físico de apariencia física con afectividad positiva $(0.23, p<.01)$ y autoconcepto físico de la competencia percibida con afectividad positiva $(0.28, p<.01)$. Secontroló por edad y por sexo, no resultando estas significativas.

\begin{tabular}{|c|c|c|c|c|c|c|}
\hline \multirow[b]{3}{*}{ Es muier } & \multicolumn{2}{|c|}{$\begin{array}{l}\text { Satisfacción con la } \\
\text { vida (SCV) }\end{array}$} & \multicolumn{2}{|c|}{$\begin{array}{l}\text { Afectividad positiva } \\
\text { (AFP) }\end{array}$} & \multicolumn{2}{|c|}{$\begin{array}{c}\text { Afectividad } \\
\text { negativa (AFN) }\end{array}$} \\
\hline & estimador & valor-p & estimador & valor-p & estimador & valor- $p$ \\
\hline & 0.25 & .07 & 0.00 & .96 & 0.14 & 0.18 \\
\hline Edad & -0.01 & 31 & 0.01 & .52 & -0.01 & 0.45 \\
\hline Nivel deAF & 0.08 & 24 & 0.09 & $.02^{*}$ & 0.02 & 0.65 \\
\hline \multicolumn{7}{|c|}{ Autoeficacia para la práctica de actividad física } \\
\hline Autoeficacia para laAF & $\begin{array}{l}0.01 \\
\text { Moti }\end{array}$ & ación & 0,15 & $.01^{*}$ & 0.14 & 0.04 \\
\hline Regulación intrínseca & 0.1 & .37 & 0.07 & .18 & 0.01 & 0.84 \\
\hline Regulación integrada & 0.02 & .84 & -0.03 & .65 & 0.07 & 0.36 \\
\hline Regulación identificada & -0.09 & .39 & 0.08 & .22 & -0.08 & 0.22 \\
\hline Regulación introyectada & -0.04 & .66 & -0.05 & .33 & 0.13 & $0.02 *$ \\
\hline Regulación externa & -0.07 & .48 & -0.06 & .4 & 0.05 & 0.53 \\
\hline Desmotivación & -0.01 & .88 & -0.08 & .2 & 0.03 & 0.66 \\
\hline \multicolumn{7}{|c|}{ Percepción de bar reras para la práctica de actividad física } \\
\hline Falta de tiempo & 0.02 & .82 & 0.01 & .89 & 0.07 & 0.37 \\
\hline Influencia social & -0.11 & .5 & 0.14 & .06 & 0.11 & 0.24 \\
\hline Falta de energía & 0.1 & 37 & 0.03 & .66 & 0.06 & 0.49 \\
\hline Falta de voluntad & -0.02 & .88 & 0 & 1 & 0.15 & $0.03 *$ \\
\hline Miedo a lastimarse & 0.22 & .09 & 0.15 & .08 & 0.17 & 0.1 \\
\hline Falta de habilidad & -0.11 & .5 & 0.08 & .37 & 0 & 0.98 \\
\hline Falta de recursos & -0.1 & .39 & -0.12 & .08 & $-0,03$ & 0.76 \\
\hline \multicolumn{7}{|l|}{ Autoconcepto físico } \\
\hline De la condición física & 0.19 & .26 & -0.03 & .74 & -0.01 & 0.89 \\
\hline De la apariencia física & 0.49 & $.00^{* * * *}$ & 0.23 & $.00 * * *$ & -0.13 & 0.13 \\
\hline De la competencia percibida & -0.02 & .88 & 0.28 & $.00 * * *$ & -0.17 & 0.07 \\
\hline De la fuerza & 0.15 & 27 & -0.08 & .31 & 0.03 & 0.71 \\
\hline De la autoestima & -0.09 & .5 & -0.03 & .75 & -0.02 & 0.8 \\
\hline
\end{tabular}

Posteriormente se probaron los modelos de media ción parcial, esto es relación de lasVSC sobre las escalas 
de bienestar subjetivo (satisfacción con la vida, afectividad positiva y afectividad negativa) mediadas por laAF. Los resultados mostraron que las relaciones entre las VSC y la satisfacción con la vida y entre la afectividad negativa, no son mediadas por el nivel deAF (coeficiente b). Esto se puede observar al analizar los intervalos de confianza que se muestran en la tabla 5, es decir que cuando el intervalo no incluye a cero, es significativo el modelo de mediación, sin embargo, en los casos de estos dos modelos, en todas sus escalas se observa que sus intervalos de confianza pasan por cero, por tanto, se concluye que no son significativos.

Respecto del efecto de cada una de lasVSC sobre la escala de afectividad positiva mediada por el nivel de $A F$, el modelo resultó significativo. Específicamente, en la tabla 6 se observa que el nivel de AF media la rela ción entre 7 VSC (autoeficacia para la práctica de AF, falta de tiempo, fal ta de energía, falta de voluntad, falta de habilidad, autoconcepto físico de la condición física, autoconcepto físico de la fuerza) y el nivel de afectividad positiva.

Tabla 5.

Mediación de las variables sociocognitivas sobre las escalas del bienestar (satiffacción con la vida, afectividad negativa, y afectividad positiva) mediada por el nivel de actividad física

\begin{tabular}{|c|c|c|c|c|c|c|c|c|c|}
\hline \multirow[t]{3}{*}{ Variables SC } & \multicolumn{9}{|c|}{ Bienestar Subjetivo } \\
\hline & \multicolumn{3}{|c|}{ Satisfacción con la vida } & \multicolumn{3}{|c|}{ Afectividad Negativa } & \multicolumn{3}{|c|}{ Afectividad positiva } \\
\hline & estimador & IC inferior & IC superior & estimador & IC inferior & IC superior & estimador & IC inferior & IC superior \\
\hline \multicolumn{10}{|c|}{ Autoeficacia para la práctica deAF } \\
\hline $\begin{array}{l}\text { Autoeficacia para la AF } \\
\text { Motivación }\end{array}$ & 0.035 & -0.024 & 0.112 & 0.009 & -0.036 & 0.055 & 0.041 & 0.006 & 0.096 \\
\hline Regulación intrínseca & 0.011 & -0.008 & 0.062 & 0.003 & -0.007 & 0.034 & 0.013 & -0.004 & 0.052 \\
\hline Regulación integrada & -0.007 & -0.061 & 0.011 & -0.002 & -0.034 & 0.007 & $-0 ., 008$ & -0.044 & 0.013 \\
\hline Regulación identificada & 0.013 & -0.007 & 0.081 & 0.003 & -0.009 & 0.040 & 0.014 & -0.003 & 0.070 \\
\hline Regulación introyectada & 0.002 & -0.020 & 0.038 & 0.001 & -0.010 & 0.021 & 0.002 & -0.023 & 0.025 \\
\hline Regulación externa & 0.013 & -0.007 & 0.065 & 0.003 & -0.011 & 0.039 & 0.015 & -0.002 & 0.056 \\
\hline Desmotivación & -0.002 & -0.041 & 0.015 & 0.000 & -0.020 & 0.008 & -0.002 & -0.027 & 0.017 \\
\hline \multicolumn{10}{|c|}{ Percepción de bar reras para la práctica deAF } \\
\hline Falta de tiempo & -0.024 & -0.097 & 0.010 & -0.006 & -0.053 & 0.021 & -0.028 & -0.077 & -0.004 \\
\hline Influencia social & 0.009 & -0.012 & 0.067 & 0.002 & -0.008 & 0.038 & 0.011 & -0.011 & 0.059 \\
\hline Falta de energía & 0.020 & -0.009 & 0.087 & 0005 & -0.018 & 0.048 & 0.023 & 0.001 & 0.080 \\
\hline Falta de voluntad & -0.035 & -0.103 & 0.027 & -0.009 & -0.061 & 0.033 & -0.040 & -0.097 & -0.004 \\
\hline Miedo a lastimarse & -0.008 & -0.076 & 0.014 & -0.002 & -0.036 & 0.008 & -0.009 & -0.060 & 0.014 \\
\hline Falta de habilidad & 0.030 & -0.013 & 0.124 & 0.008 & -0.027 & 0.062 & 0.034 & 0.004 & 0.105 \\
\hline Falta de recursos & -0.015 & -0.096 & 0.007 & -0.004 & -0.051 & 0.010 & -0.017 & -0.064 & 0.004 \\
\hline \multicolumn{10}{|l|}{ Autoconcepto físico } \\
\hline De la condición física & 0.045 & -0.026 & 0.165 & 0.012 & -0.044 & 0.083 & 0.052 & 0.06 & 0.133 \\
\hline De la apariencia física & -0.009 & -0.065 & 0.011 & -0.002 & -0.038 & 0.008 & -0.011 & -0.052 & 0.014 \\
\hline De la competencia percibida & 0.004 & -0.020 & 0.067 & 0.001 & -0.010 & 0.029 & 0.004 & -0.023 & 0.053 \\
\hline Dela fuerza & -0.030 & -0.106 & 0.019 & -0.008 & -0.059 & 0.030 & $-0,035$ & -0.097 & -0.004 \\
\hline De la autoestima & 0.010 & -0.013 & 0.069 & 0.003 & -0.009 & 0.045 & 0.012 & -0.010 & 0.064 \\
\hline Total & 0.062 & -0.034 & 0.214 & 0.016 & -0.066 & 0.108 & 0.071 & 0.010 & 0.187 \\
\hline
\end{tabular}

\section{Discusión}

En este estudio lasVSC que resultaron predictoras del nivel de AF, fueron: (1) autoeficacia para la $A F,(2)$ falta de tiempo, (3) falta de voluntad, (4) falta de habilidad, (5) autoconcepto de condición física, y (6) autoconcepto físico de fuerza. Esto es consistente con estudios previos que han mostrado que una baja autoeficacia, y alta percepción de barreras explican la disminución de AF (Van Dyck, De Bourdeaudhuij, Deliens \& Deforche, 2015).
Respecto de la autoeficacia, estos hallazgos son concordantecon un estudio (Alpkaya, 2019) que determinó la relación entre autoeficacia para laAF y el nivel deAF en estudiantes, encontrando una relación significativa y positiva. También coincide con los resultados de un metaanál isis que concluyó que la autoeficacia para laAF no sólo se relaciona a la práctica deAF, sino también es un predictor del comportamiento futuro (Tang, Smith, Mc Sharry, Hann \& French, 2018).

Respecto de la percepción de barreras, este estudio confirma su influencia en la práctica de AF, mostrando que, a mayor falta de tiempo y falta de voluntad, menor nivel deAF, y a mayor fal ta de habilidad, mayor nivel de AF. En el caso de la barrera falta de tiempo, un estudio longitudinal (Van Dyck et al., 2015) mostró que las ba rreras relacionadas con el tiempo predijeron una disminución de la práctica de AF. En el caso de la barrera falta de voluntad un estudio la identificó como la barrera más frecuente para la práctica de AF (Gupta $\&$ Varghese, 2019). En definitiva, resultados de estudios previos concuerdan que las principales barreras son la falta de tiempo y la falta de voluntad en quienes registran niveles bajos deAF (Rubio-Henao, Correa \& Ramírez-Vélez, 2015). Finalmente, la falta de habilidad, aunque es considerada una de las barreras para la práctica de AF, los resultados de este estudio no lo confirman. Sin embargo, hallazgos similares se han evidenciado (Alcázar, Yamalis, Lobo \& M endinueta, 2016) donde establecen una relación negativa, es decir, menor habilidad a mayor práctica de AF en estudiantes. Una hipótesis explicativa es que, aquellos estudiantes que tienen la percepción de no ser hábiles para laAF dedican mayor práctica de AF para revertir esa situación (Rodríguez, Valencia, Gaitán, González \& León, 2017).

Respecto del autoconcepto físico, los resultados mostraron que este influye en la AF, específicamente, a mayor nivel de autoconcepto de condición física, mayor nivel deAF y que a mayor autoconcepto físico de fuerza, menor nivel deAF. Es importante mencionar que la investigación previa ha demostrado una relación positiva entre las diferentes dimensiones del autoconcepto y los niveles de AF (O netti- $O$ netti, Chinchilla-M inguet, Lourenço \& Castillo-Rodriguez, 2019). Particularmente la relación significativa, pero indirecta encontrada en la presente investigación (a mayor nivel de autoconcepto físico de fuerza, menor nivel de AF), también ha sido 
encontrada en otra investigación (Fernández-Bustos, Infantes-Paniagua, Cuevas\& Contreras, 2019). Además, este estudio estableció que a mayor autoconcepto de condición física, mayor nivel deAF, resultado similar al de investigaciones previas (Guillamón, Canto \& López, 2019).

Entre las variables propuestas como predictoras de la AF y que no resultaron significativas, está la motivación. Hallazgos similares se encontraron en un estudio donde sólo la regulación integrada fue predictora de la AF moderada vigorosa y la AF total, mientras que la desmotivación, regulación externa, regulación introyectada, regulación identificada y motivación intrínseca, no fueron predictores (Gómez, Sánchez-O liva $\&$ Labisa, 2020). Así también, un metaanálisis advierte que, si bien sus hallazgos proporcionan cierto apoyo a los principios de la teoría de la autodetermina ción, estos mostraron heterogeneidad sustancial en la mayoría de las asociaciones y muchos estudios tuvieron deficiencias metodológicas, agregando además que, incluso los efectos más fuertes observados fueron sólo de tamaño débil a moderado. Lo anterior podría sugerir que otros factores podrían ser predictores importantes de los niveles de AF (O wen, Smith, Lubans, $\mathrm{Ng} \&$ Lonsdale, 2014). Así, una posible explicación del resultado de este estudio podría ser que el modelo no sólo integró la variable motivación para predecir la $A F$, sino variasVSC, y por tanto desaparece su efecto en el modelo.

Respecto del análisis del efecto de mediación del nivel de AF entre lasVSC y el bienestar subjetivo, los resultados mostraron que, de las tres escalas de bienestar, laAF sólo tiene un efecto mediador entre variables sociocognitivas y la afectividad positiva. Además, cuando se analizó el efecto de cada una de lasVSC, se encontró que las variables (1) autoeficacia para la práctica de $A F$, (2) falta de tiempo, (3) falta de energía, (4) falta de voluntad, (5) falta de habilidad, (7) autoconcepto físico de la condición física, (7) autoconcepto físico de la fuerza tuvieron un efecto de mediación significativo sobre la afectividad positiva medidas por el nivel de AF. Resultados similares se encontraron en un estudio en universitar ios que mostraron que laAF tuvo efectos directos positivos sobre laautoeficacia paralaAF, la autoestima física, afecto positivo y afecto negativo; además, que la autoestima física fue la variable mediadora más poderosa en la calidad de vida, seguida por el afecto positivo y el afecto negativo (Joseph, Royse, Benitez \& Pekmezi, 2014). Además, se considera que los resultados del presente estudio aportan a la investigación previa, que se ha centrado en la relación directa entre la AF y el bienestar, no integrando otras variables predictoras, y al mismo tiempo a la AF como mediadora (W iese, Kuykendall \&Tay, 2018).

Las limitaciones de esta investigación que podrían dificultar la generalización de resultados, y necesarias de considerar al interpretar los resultados son tres: la representatividad de la muestra, este estudio consideró una muestra no probabilista por conveniencia, de estudiantes a los cuales se tenía acceso; el Diseño Predictivo Transversal, el cual no suele emplear ningún procedimiento de control de variables extrañas y su diseño contempla una medida única, por tanto, es difícil derivar las relaciones causal es y es propenso a ciertos sesgos, lo cual implica tener cuidado al interpretar las asociaciones y la dirección de las asociaciones; y el IPAQ como instrumento de medida de laAF podría haber implica do limitaciones relacionadas con el llamado sesgo del recuerdo puesto que exige informar de modo diferenciado los tiempos destinados a distintos tipos de actividades en los últimos 7 días, y además los sesgos propios de instrumentos de auto reporte.

En futuras investigaciones se sugiere emplear técnicas de muestreo probabilístico, un diseño longitudinal y otros instrumentos objetivos de medida deAF que permitan responder a la complejidad del constructo y dar cuenta con exactitud de su nivel en las personas, no sobre ni subestimándola (M ullen \& Conroy 2019). Esimportante avanzar no sólo en el estudio de las relaciones de las diferentes variables que predicen, median o moderan el nivel de práctica de AF; sino ir más allá en el diseño e implementación de intervenciones que consideren lasVSC que han mostrado relaciones significativas con la AF (Rhodes, Janssen, Bredin, Warburton $\&$ Bauman, 2017), ampliando también a otros niveles educativos considerando la necesidad de desplegar intervenciones en etapas previas a la universidad (Giakoni, Bettancourt \& Duclos-Bastías, 2021).

\section{Conclusión}

En base a los resultados y discusión presentada en esta investigación, se concluye: (1) la autoeficacia para laAF, el autoconcepto físico, y la percepción de barreras para la AF predicen el nivel de AF; además (2) el nivel de AF tiene un efecto de mediación entre el conjunto deVSC y la afectividad positiva. Específicamente, el nivel de AF media la relación entre la autoeficacia para la práctica de $A F$, falta de tiempo, falta de energía, falta de voluntad, falta de habilidad, autoconcepto físico 
de la condición física, autoconcepto físico de la fuerza con afectividad positiva.

Este estudio proporcionó un análisis de los factores sociocognitivos relacionados al nivel de práctica de AF en universitarios. También proporcionó información detallada sobre la mediación de la AF entreVSC y el bienestar subjetivo. Se considera un aporte al poner a disposición un estudio sobre variables claves relaciona das a la práctica de AF y un insumo importante para desarrolladores de programas que buscan mejorar los niveles de AF en esta etapa clave para el establecimiento de conductas de salud. Así, considerando las escasas inter venciones en educación superior, se invita a considerar lasVSC predictoras de la AF en la generación de iniciativas institucionales que permitan su desarrollo, para aumentar los niveles de AF del estudiantado y consecuentemente su bienestar. Se recomienda el diseño de estos programas integrados idealmente al currículum de las carreras (M ellaN orambuena, Celis, SáezDelgado, Aeloiza, Echeverria, Nazar \& PetermannRocha, 2019).

\section{Agradecimientos}

Este estudio fue financiado con la BecaA poyo Rea lización de Tesis 2019, Programa Magíster en Psicología, Universidad de Concepción, Chile.

\section{Referencias}

Alcázar, M., Yamalis, J., Lobo, D., \& Mendinueta, M. (2016). Nivel de Actividad Física en Trabajadores de una Empresa de Transporte Terrestre en la Ciudad de Barranquilla. Revista Salud En Moviemientomiento, 8(1), 1-13.

Alpkaya, U. (2019). The relationship between the physical activity efficacy and physical activity of the middle school students. Pedagogics, Psychology, M edical-Biological Problems of Physical Training and Sports, 23(2), 59-65. doi: 10.15561/ 18189172.2019.0202

Álvarez, C., Briceño, A., Álvarez, K., Abufhele, M., \& Delgado, I. (2018). Estudio de adaptación y valida ción transcultural de una escala de satiffacción con la vida para adolescente. Revista Chilena de Pediatria, 89 (1), 51-58. doi: 10.4067/ S037041062018000100051

Ato, M. , López, J. , \& Benavente, A. (2013). Un sistema de clasificación de los diseños de investigación en Psicología. Analesde Psicologia, 29 (3), 1038-1059. doi: 10.6018/ analesps. 29.3.178511
Breen, R., Karlson, K., \& Holm, A. (2018). Interpreting and understanding logits, probits, and other nonlinear probability models. Annual Review of Sociology, 44, 3954.

Carrera, Y. (2017). Cuestionario Internacional de Actividad Física (IPAQ). Revista Enfermeria Del Trabajo, 7(11), 49-54. doi: 10.1016/ j.nefrol.2016.11.002

Chanal, J., Cheval, B., Courvoisier, D., \& Paumier, D. (2019). Developmental relations between motivation types and physical activity in elementary school children. Psychology of Sport and Exercise, 43, 233-242.

Corella, C., Rodríguez-M uñoz, S., Abarca-Sos, A., \& Zaragoza, J. (2018). Cumplimiento de las recomendaciones de práctica de actividad física en función de los cutofs points y el género en estudiantes universitarios españoles. Revista Euroamericana de Ciencias D el Deporte, 7(1), 9- 18.

Deci, E., \& Ryan, R. (2000). The» what» and» why» of goal pursuits: Human needs and the selfdetermination of behavior. Psychological Inquiry, 11(4), 227-268.

Delgado, M., Zamarripa, J., De La Cruz, M., CantúBerrueto, A. \& \& Álvarez, O. (2017). Validación de la versión mexicana del Cuestionario de Auto-eficacia parael Ejercicio. Revista dePsicologia D el Deporte, 26(2), 85-90.

Diener, E. (1984). Subjective Well-being. Psychological Bulletin, 95(3), 542-575. doi: 10.1002/ 9781118339893. wbeccp518

Dufey, M., \& Fernandez, A. (2012). Validez y confiabilidad del positive affect and negative affect schedule (PANAS) en estudiantes univer sitarios chilenos. Revista Iberoamericana de Diagnóstico y Evaluación, 2(34), 157-173.

Efron, B. (1983). Estimating the error rate of a prediction rule: Improvement on cross-validation. Journal of the American Statistical Association, 78(382), 316-331. doi:10.1080/ 01621459.1983.10477973

Fernández-Bustos, J., Infantes-Paniagua, Á., Cuevas, R., $\&$ Contreras, O. (2019). Effect of physical activity on self-concept:Theoretical model on the mediation of body image and physical self-concept in adolescents. Frontiers in Psychology, 10(7), 1537. doi:10.3389/ fpsyg.2019.01537

Fox, K., \& Corbin, C. (1989). The Physical SelfPerception Profile: Devlopment and Preliminary Validation. Journal of Sport and Exercise Psychology, 11(4), 408-430. doi:10.1111/ j.1464410X.1989.tb05254.x 
Giakoni, F. , Bettancourt, P., \& Duclos-Bastías, D. (2021). Educación Física en Chile: tiempo de dedicación y su influencia en la condición física, composición corporal y nivel de actividad física en escolares. Retos, (39), 24-29.

Gómez, M., Sánchez-O liva, D., \& Labisa, A. (2020). Actividad física en tiempo libre en estudiantes universitarios colombianos. Retos, 37(1), 181-189.

González-Cutre, D., Sicilia, Á., \& Fernández, A. (2010). Hacia una mayor comprensión de la motivación en el ejercicio físico: medición de la regulación integradaen el contexto español. Psichothema, 22(4), 841847.

Guillamón, A., Canto, E. \& López, P. (2019). Actividad física, condición física y autoconcepto en escolares de 8 a 12 años. Retos, 2041(35), 236-241.

Gupta, G. \& \&arghese, P. (2019). Self Reported Physical Activity Level and Barriers to Physical Activity in Youth of Bangalore, India. Journal of Clinical and Diagnosic Research, 13(10), 10-13. doi: 10.7860/ jcdr/ 2019/42281.13243

Joseph, R., Royse, K ., Benitez,T., \& Pekmezi, D. (2014). Physical activity and quality of life among university students: exploring self-efficacy, self-esteem, and affect as potential mediators. Quality of Life Research, 23(2), 659-667. doi:10.1007/ s11136-013-0492-8

Kim, Y., Lumpkin, A., Lochbaum, M., Stegemeier, S., $\&$ Kitten, K. (2018). Promoting physical activity using a wearable activity tracker in college students: A cluster randomized controlled trial. Journal of Sports Sciences, 36(16), 1889-1896. doi:10.1080/ 02640414.2018.1423886

Lemoyne, J., Valois, P., \& Guay, F. (2015). Physical selfconcept and participation in physical activity in college students. Medicine and Science in Sports and Exercise, 47(1), 142-150. doi:10.1249/ MSS.0000000000000378

Lubans, D., O kely, A., M organ, P., Cotton, W., Puglisi, L., \& Miller, J. (2012). Description and evaluation of a social cognitive model of physical activity behaviour tailored for adolescent girls. Health Education Research, 27(1), 115-128.

M ajeed, N., Jabbar, M., \& J un, X. (2017). Social Cognitive Factors Associated W ith Physical Activity among U niversity Students: A C ross-Sectional Study. M 0], 2(2), 00017.

Marcus, B., Selby, V., Niaura, R., \& Rossi, J. (1992). Self-efficacy and the stages of exercise behavior change. Research Q uarterly for Exercise and Sport, 63(1), 60-66. doi: 10.1080/ 02701367.1992.10607557
Martínez-Lemos, R., Puig, A., \& García-García, 0. (2014). Perceived barriers to physical activity and related factors in Spanish university students. Open Journal of Preventive M edicine, 4, 164-174

M ella-Norambuena, J., Celis, C., Sáez-D elgado, F., Aeloiza, A., Echeverria, C., Nazar, G., \& PetermannRocha, F. (2019). Revisión sistemática de práctica de actividad física en estudiantes universitarios. Re vista Iberoamericana de Ciencias de la Actividad Física y el Deporte, 8(2), 37-58.

M irzaei-Alavijeh, M., Soroush, A., Nasirzadeh, M., Hatamzadeh, N., Zinat-M otlagh, F., Jalilian, F., ... Mahboubi, M. (2018). Socio-Cognitive determinants of regular physical activity among college students. Middle East Journal of Family M edicine, 16(2), 158-162. doi: 10.5742/ MEW FM.2018.93256

Moreno, J., y Cervelló, E. (2005). Physical selfperception in Spanish adolescents: effects of gender and involvement in physical activity. Journal of H uman M ovement Studies, 48, 291-311.

Mullen, S. \& Conroy, D. (2019). InnovativeA pplications of Technology for theAssessment and Promotion of Physical Activity. Psychology of Sport and Exercise, 41(19), 144-145. doi: 10.1016/ j. psychsport.2019.01.004

OMS. (2010). Recomendaciones mundiales sobre laactividad física para la salud. World $H$ ealth O rganization, 1-58.

O netti-O netti, W., Chinchilla-M inguet, J., Lourenço, F., \& Castillo-Rodriguez,A. (2019). Self-concept and physical activity: Differences between high school and university studentsin Spain and Portugal. Frontiers in Psychology, 10(6), 1333. doi: 10.3389/ fpsyg. 2019.01333

O wen, K., Smith, J., Lubans, D., N g, J., \& Lonsdale, C. (2014). Self-determined motivation and physical activity in children and adolescents: A systematic review and meta-analysis. PreventiveM edicine, 67, 270279.

Pereira, L., Fernandez, E., Cruz., \& Santiesteban, J. (2018). Programa de actividad física y su incidencia en la depresión y bienestar subjetivo de adultos mayores. Retos(33), 14-19.

Ramírez-Vélez, R., Triana-Reina, H., Carrillo, H., \& Ramos-Sepúlveda, J. (2016). Percepción de barreras para la práctica de la actividad física y obesidad abdominal en universitarios de Colombia. Nutrición Hospitalaria, 33(6), 1317-1323. doi: http:/ / dx. doi.org/ 10.20960/ nh.574

Rhodes, R., Janssen, I., Bredin, S., Warburton, D., \& 
Bauman, A. (2017). Physical activity: Health impact, prevalence, correlates and interventions. Psychology and $H$ ealth, 32(8), 942-975. doi: 10.1080/ 08870446.2017.1325486

Rodríguez, F., Valencia, S., Gaitán, E., González, S., \& León, J. (2017). Hábitos saludables, motivos y ba rreras en la realización de actividad física en estudiantes universitarios. Revista de Investigación: Cuerpo, Cultura y Movimiento, 7(1), 81-102.

Rubio-Henao, R., Correa, J., \& Ramírez-Vélez, R. (2015). Propiedades psicométricas de la versión al Español del cuestionario «barriers to being active quiz», entre estudiantes universitarios de Colombia. Nutricion Hospitalaria, 31(4), 1708-1716. doi: 10.3305/ nh.2015.31.4.8404

Serón, P., M uñoz, S., \& Lanas, F. (2010). Nivel de actividad física medida a través del cuestionario internacional de actividad física en población chilena. Re vista M edica deChile, 138, 1232-1239. doi: 10.4067/ S0034-98872010001100004

Sevil, J., Práxedes, A., Zaragoza, J., Del Villar, F., \& Garcia-González, L. (2017). Barreras percibidaspara la práctica de actividad física en estudiantes universitarios. Diferencias por género y niveles de actividad física. U niversitas Psychologica, 16(4), 1-15.

Sibley, B., \& Bergman, S. (2016). Relationships among goal contents, exercise motivations, physical activity, and aerobic fitness in university physical education courses. Perceptual and M otor Skills, 122(2), 678-700. doi: $10.1177 / 0031512516639802$

Strecher, V., M cEvoy, B., Becker, M., \& Rosenstock, I. (1986). The role of self-efficacy in achieving health behavior change. Health Education Quarterly, 13(1), 73-92.

Tang, M., Smith, D. , M c Sharry, J., Hann, M ., \& French, D. (2018). Behavior change techniques associated with changes in postintervention and maintained changes in self-efficacy for physical activity: A systematic review with meta-analysis. Annals of Behavioral Medicine, 53(9), 801-815. doi: 10.1093/ abm/ kay090

Van Dyck, D., De Bourdeaudhuij, I., Deliens, T., \& Deforche, B. (2015). Can Changes in Psychosocial Factors and Residency Explain the Decrease in Physical Activity During the Transition from High School to College or U niversity? International Journal of Behavioral M edicine, 22(2), 178- 186. doi: 10.1007/ s12529-014-9424-4

Warburton, D., \& Bredin, S. (2017). Health benefits of physical activity: a systematic review of current systematic reviews. Current Opinion in Cardiology, 32(5), 541-556.

W iese, C., Kuykendall, L. \& \& Tay, L. (2018). Get active? A meta-analysis of leisure-time physical activity and subjective well-being The ournal of Positive Psychology, 13(1), 57-66.

W illiams, S., \& French, D. (2011). W hat are the most effective intervention techniques for changing physical activity self-efficacy and physical activity behaviour-and are they the same? Health Education Research, 26(2), 308-322. doi:10.1093/ her/ cyr005

Xu, J., Bauldry, S., \& Fullerton, A. (2019). Bayesian approaches to assessing the parallel lines assumption in cumulative ordered Logit Models. Sociological Methods \& Research, 1(1), 1-32. doi: 10.1177/ 0049124119882461

Young, M., Plotnikoff, R., Collins, C., Callister, R., \& Morgan, P. (2014). Social cognitive theory and physical activity: a systematic review and meta analysis. Obesity Reviews, 15(12), 983-995.

Zhang, Z., He, Z., \& Chen, W. (2020). The relationship between physical activity intensity and subjective well-being in college students. Journal of American College Health, 1-6. doi: 10.1080/ 07448481.2020 .1790575

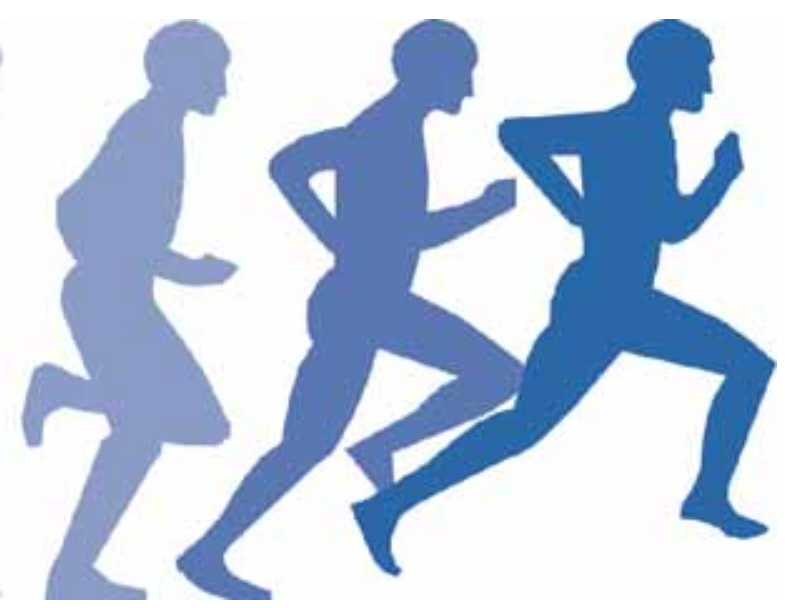

